\title{
Inhibition of miR-128-3p Attenuated Doxorubicin-Triggered Acute Cardiac Injury in Mice by the Regulation of PPAR- $\gamma$
}

\author{
Wen-Bin Zhang ${ }^{(D)}$, Yong-Fa Zheng $(\mathbb{D}$, and Yao-Gui Wu \\ Department of Cancer Center, Renmin Hospital of Wuhan University, Wuhan, Hubei 430060, China \\ Correspondence should be addressed to Yao-Gui Wu; wuyaoguitougao@163.com
}

Received 8 June 2020; Revised 26 March 2021; Accepted 31 March 2021; Published 20 April 2021

Academic Editor: Antonio Brunetti

Copyright ( 2021 Wen-Bin Zhang et al. This is an open access article distributed under the Creative Commons Attribution License, which permits unrestricted use, distribution, and reproduction in any medium, provided the original work is properly cited.

\begin{abstract}
Background. The clinical usefulness of doxorubicin (DOX), an anthracycline with antitumor activity, is limited by its cardiotoxicity. Oxidative stress and myocardial apoptosis were closely associated with DOX-induced cardiac dysfunction. It has been reported that microRNA-128-3p (miR-128-3p) was involved into the regulation of redox balance. However, the role of miR-128-3p in DOXrelated cardiac injury remains not yet understood. The aim of this study was to investigate the biological effect of miR-128-3p in DOX-induced cardiotoxicity. Methods. To induce DOX-related acute cardiac injury, mice were subjected to a single injection of DOX. Inhibition of myocardial miR-128-3p was achieved by an adeno-associated virus (AAV9) system carrying a miR-128-3p sponge. Results. The data in our study indicated that miR-128-3p was upregulated in DOX-treated hearts and cardiomyocytes. Inhibition of miR-128-3p attenuated DOX-related cardiac injury and improved cardiac function in mice. Moreover, miR-128-3p inhibition could suppress myocardial inflammatory response, oxidative damage, and cell apoptotic death in DOX-treated mice. Further analysis showed that miR-128-3p could directly target peroxisome proliferator-activated receptor $\gamma$ (PPAR- $\gamma$ ) and decrease PPAR- $\gamma$ expression. Moreover, the protective effects provided by miR-128-3p inhibition were abolished by a PPAR- $\gamma$ antagonist in vivo and in vitro. Conclusions. miR-128-3p inhibition attenuated DOX-related acute cardiac injury via the regulation of PPAR- $\gamma$ in mice.
\end{abstract}

\section{Introduction}

Doxorubicin (DOX) is an effective antitumor anthracycline antibiotic. Despite its clinical efficacy, the use of DOX is associated with a progressive cardiomyopathy that leads to congestive heart failure $[1,2]$. It has been estimated that about $21 \%$ of patients developed chemotherapy-related cardiotoxicity after DOX treatment [3]. Currently, there are no drugs that can block the occurrence of DOX-related cardiac injury.

Multiple factors have been reported to be involved in the pathogenesis of DOX-induced cardiotoxicity. Of them, excessive oxygen species (ROS) production and subsequent myocardial apoptotic cell death were the main mediators of DOX-induced cardiotoxicity $[1,4]$. Excessive ROS induced oxidative damage to biological macromolecules and disrupted cellular membrane integrity [5]. In addition, DOX treatment resulted in the release of cytochrome $\mathrm{c}$ and thus causing caspase- 3 activation and apoptosis [6]. Thus, finding approaches to prevent DOX-related myocardial ROS pro- duction and apoptosis would be of great significance for the treatment of DOX-induced cardiac injury.

MicroRNAs (miRNAs) are single-stranded noncoding RNAs that can regulate genes at the posttranscriptional level [7]. Accumulating evidences suggested that miRNAs were closely involved into DOX-induced cardiotoxicity [8]. It has been reported that miR-128-3p was a tumor suppressor and could inhibit metastasis of esophageal squamous-cell cancer [9]. miR-128-3p was closely involved into the occurrence of oxidative stress and myocardial inflammation induced by lipopolysaccharide [10]. Moreover, inhibiting miR-128-3p expression suppressed apoptosis of cardiomyocytes in response to ischemia/reperfusion [11]. However, the role of miR-128-3p in DOX-induced cardiotoxicity is still unknown. Here, we clearly showed that the inhibition of miR-128-3p ameliorated DOX-induced cardiomyocyte injury and cardiac dysfunction, which correlated with the reduction in oxidative damage, inflammatory response, and myocardial apoptosis in mice. 


\section{Methods}

2.1. Animals and Models. A total of 48 C57BL/6 (weight: 22$26 \mathrm{~g}$ ) were purchased from HFK Bioscience (Beijing, China) and housed in the specific-pathogen-free environment of Renmin Hospital of Wuhan University. These mice were randomly separated into four groups ( $n=12$ each group): normal saline (NS)+control, NS+miR-128-3p sponge, $\mathrm{DOX}+$ control, and DOX $+\mathrm{miR}-128-3 \mathrm{p}$ sponge. The adenoassociated virus (AAV9)-U6-GFP vectors carrying miR128-3p sponge or a negative control were generated by Genecopia (Shanghai, China). AAV9-miR-128-3p sponge, which was dissolved into $50 \mu \mathrm{l}$ PBS, was injected via the tail vein at a dose of $5 \times 10^{11}$ viral genome particles per mouse $[12,13]$. Three weeks later, after the efficiency of this sponge was evaluated, the mice received an intraperitoneal injection of DOX (purity $\geq 98 \%$, Novopharm, $15 \mathrm{mg} / \mathrm{kg}$ ). An equal volume of NS was administered to mice as a control. All the mice were observed for 3 days. After that, the mice were subjected to the hemodynamics. To confirm the role of peroxisome proliferator-activated receptor $\gamma(\operatorname{PPAR}-\gamma)$ in the protection provided by miR-128-3p inhibition, mice were treated with a PPAR- $\gamma$ inhibitor (GW9662, $0.35 \mathrm{mg} / \mathrm{kg}$ per day in drinking water) for 10 days beginning at one week before DOX injection as previously described [14]. This study was reviewed and approved by the Institutional Animal Care and Use Committee at Renmin Hospital of Wuhan University.

2.2. Hemodynamics. Mice were anesthetized with $2 \%$ isoflurane, and a microtip catheter transducer was inserted into the left ventricle; the signals were recorded by a Millar PressureVolume system (Millar, Inc.) [15]. Parameters including maximal slope of the systolic pressure increment $(+\mathrm{dP} / \mathrm{dtmax})$ and diastolic pressure decrement (-dP/dt max), ejection fraction (EF), and left ventricular end-diastolic pressure (LVEDP) were analyzed.

2.3. Cardiac Injury Assessment, Measurement of Inflammatory, and Oxidative Markers. Blood samples were collected from the mice to detect cardiac troponin I (cTnI) and N-terminal probrain natriuretic peptide (NT-proBNP) for the evaluation of cardiac injury. Mouse NT-proBNP (\#CSB-E05153m) kit and cTnI ELISA kit (\#CSB-E08421m) were obtained from CUSABIO (Wuhan, China).

The superoxide dismutase (SOD) activity assay kit, catalase activity kit, glutathione peroxidase (Gpx) activity kit, and glutathione (GSH) content assay kit were all purchased from Naijing Jiancheng Bioengineering Institute (Nanjing, China). Malondialdehyde (MDA) assay kit was used to detect MDA in the supernatant via thiobarbituric acid method according to the instructions. 4-Hydroxynonenal (4-HNE)-protein adducts detection kit was provided by Abcam (\#ab238538). 3-Nitrotyrosine (3-NT) competitive ELISA was provided by Abcam (\#ab113848).

DNA-p65 nuclear factor kappa-B (NF- $\kappa \mathrm{B})$ binding assay was detected with a Mercury TransFactor kit (BD Biosciences, Clontech). To detect myocardial inflammatory response, fresh heart samples were homogenized to detect myocardial tumor necrosis factor (TNF) $-\alpha$ and interleukin
(IL)-6 expression. TNF- $\alpha$ Mouse ELISA Kit and IL-6 ELISA Kit were provided by R\&D Systems.

2.4. Quantitative Real-Time PCR. The heart samples were lysed by TRIzol reagent, and the total RNA was collected and reverse-transcribed using a PrimeScript RT reagent kit (Takara Bio, Inc., Otsu, Japan). After that, the PCR amplifications were quantified using the SYBR ${ }^{\circledR}$ Green Master Mix kit (Takara Bio, Inc.). We used glyceraldehyde-3-phosphate dehydrogenase (GAPDH) as the internal control. miR-1283 p level was reverse-transcribed using miScript II RT Kit (Qiagen, Valencia, USA) and then quantified by real-time RT-PCR using the miScript SYBR green PCR kit. U6 were used as internal controls for miRNA. The specific primer for miR-128-3p is GGTCACAGTGAACCGGTC (sense) and GTGCAGGGTCCGAGGT (antisense).

2.5. Western Blotting. The total proteins were extracted from frozen hearts, and protein samples were separated by $10 \%$ SDS-PAGE and then transferred onto a polyvinylidene difluoride membrane (Millipore) [16]. The membranes were incubated overnight at $4{ }^{\circ} \mathrm{C}$ with the following primary antibodies: phospho-p65 (ab16502, 1: 1000 dilution), proliferating cell nuclear antigen (PCNA, ab92552, $1: 1000$ dilution), Bax (ab32503, 1:1000 dilution), PPAR- $\gamma$ (ab45036, 1:1000 dilution), Histone H3 (ab176842, 1:1000 dilution), and GAPDH (ab181602, 1:1000 dilution). These primary antibodies were obtained from Abcam (Shanghai, China). After incubation with secondary antibodies, the specific bands were visualized by an ECL detection system. We used GAPDH as the internal control.

2.6. Cell Culture and Treatment. Primary neonatal rat cardiomyocytes (NRCMs) were prepared according to as a previous study [13]. These cells were cultured in Dulbecco's modified Eagle's medium (HyClone; GE Healthcare Life Sciences, Logan, UT, USA) supplemented with $10 \%$ fetal bovine serum (Thermo Fisher Scientific, Inc., Waltham, MA, USA) in an incubator at $37^{\circ} \mathrm{C}$ with $5 \% \mathrm{CO}_{2}$ under a humidified atmosphere for 48 hours. After that, the cells were transfected with $20 \mu \mathrm{mol} / 1$ of miR-128-3p mimics or negative control (NC) by using a FuGENE HD reagent (Roche). To inhibit miR-128-3p in cardiomyocytes, the cells were transfected with $20 \mu \mathrm{mol} / \mathrm{l}$ of a miR-128-3p inhibitor. To verify PPAR- $\gamma$ was involved into the protection by miR-128-3p inhibition, cells were given a specific PPAR- $\gamma$ antagonist (GW9662, $10 \mu \mathrm{mol} / \mathrm{l})$. To perform luciferase reporter assay, $3^{\prime}$-untranslated regions ( $3^{\prime}$-UTR) of PPAR- $\gamma$ was inserted into pmirGLO vector, which was obtained from Promega. The cells were seeded in 24-well plates and transfected with a miR-128-3p mimic $(0.4 \mu \mathrm{g})$ or negative control with $3^{\prime}$-UTR of PPAR- $\gamma$ vector $(0.4 \mu \mathrm{g})$ using a FuGENE HD reagent (Roche). After 48 hours, the luciferase activity was detected using the Dual Luciferase Reporter Assay System (Promega). Cell viability was assessed by a cell counting kit (CCK-8 kit) followed the manufacturer's instructions.

2.7. The Detection of Intracellular ROS. The intracellular ROS production was assessed using a probe called $2^{\prime}, 7^{\prime}$ 


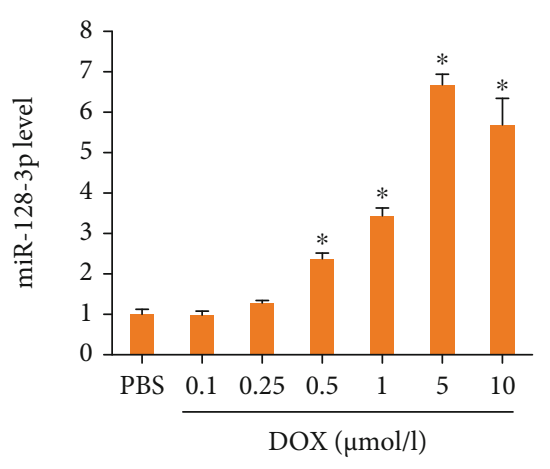

(a)

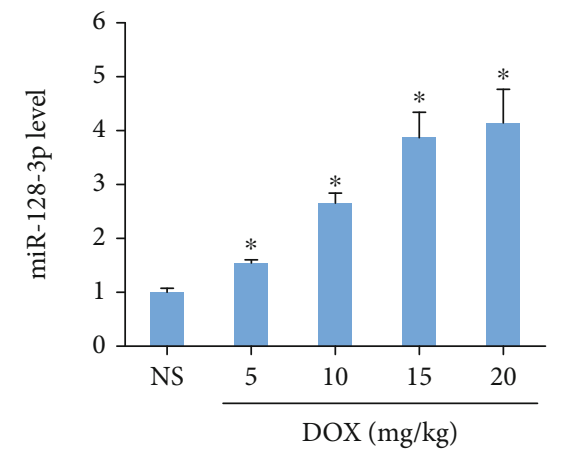

(c)

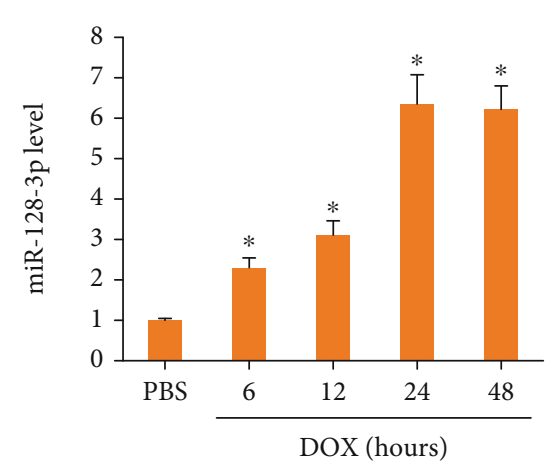

(b)

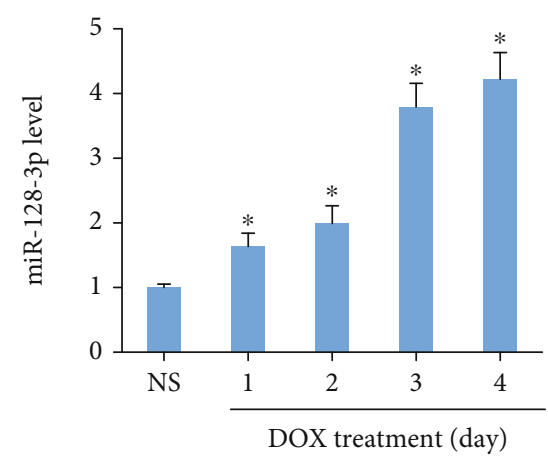

(d)

FIgURE 1: DOX decreased miR-128-3p expression. (a, b) The level of miR-128-3p expression in DOX-treated cells ( $n=6$ for each points). (c, d) The level of miR-128-3p expression in DOX-treated hearts ( $n=6$ for each points). Data are expressed as mean \pm SEM. ${ }^{*} P<0.05$ vs. NS or PBS group.

-dichlorodihydrofluorescein diacetate (DCFH-DA). The cells were cultured in a 6-well plates for 48 hours and then incubated with DCFH-DA $(10 \mu \mathrm{mol} / \mathrm{l})$ for $30 \mathrm{~min}$. The intensity of this probe was observed under a fluorescence microscope (OLYMPUS, Tokyo, Japan) [17, 18].

2.8. Apoptosis Assessment. To evaluate myocardial apoptosis in DOX-treated mice, the heart sections were dehydrated and subjected to terminal deoxynucleotidyl transferasemediated dUTP nick end-labeling (TUNEL) staining using a commercially available kit. Caspase- 3 activity in myocardial tissues was measured with a caspase- 3 colorimetric protease assay according to the manufacturer's instructions.

2.9. Statistics Analysis. All values are expressed as mean \pm SEM. Differences among groups were determined by oneway ANOVA followed by the post hoc Turkey test. Comparisons between two groups were performed by using the unpaired Student's $t$-test. $P<0.05$ was considered to be statistically significant.

\section{Results}

3.1. miR-128-3p Was Upregulated in DOX-Treated Hearts and Cardiomyocytes. To elucidate the biological function of miR-128-3p during DOX-related cardiac injury, we first detected miR-128-3p expression in cardiomyocytes, and we found that DOX dose-dependently increased the expression of miR-128-3p (Figure 1(a)). DOX also time-dependently upregulated the expression of miR-128-3p in cardiomyocytes (Figure 1(b)). Next, we detected expression of miR-128-3p in DOX-treated hearts and found that DOX dose- and timedependently increased miR-128-3p expression in heart samples (Figures 1(c) and 1(d)). These results indicated that miR128-3p expression was remarkably increased in DOX-treated hearts, suggesting an involvement of miR-128-3p in DOXtreated hearts.

3.2. miR-128-3p Inhibition Alleviated Cardiac Injury and Improved Cardiac Function in DOX-Treated Mice. Here, we used AAV9 system carrying a miR-128-3p sponge to inhibit miR-128-3p expression in the hearts. The data in our study demonstrated that this sponge significantly decreased miR128-3p expression in the hearts (Figure 2(a)). Moreover, with this sponge infection, miR-128-3p expression in DOX-treated hearts almost declined to the normal level (Figure 2(a)). DOX significantly decreased body weight and the ratio of body weight to tibial length; however, the two were normalized in mice with miR-128-3p inhibition (Figures 2(b) and 2(c)). miR-128-3p inhibition also restored the ratio of heart weight to tibial length to the normal level (Figure 2(d)). Furthermore, the markers of cardiac injury, including BNP mRNA level, cTnI, and NT-proBNP were increased in DOX-treated mice but decreased in mice with DOX + miR-128-3p sponge (Figures 2(e)-2(g)). Next, we detected cardiac function in DOX-treated mice. We found that miR-128-3p inhibition had no profound effects on the decreased heart rate in DOX-treated mice (Figure 3(a)). 


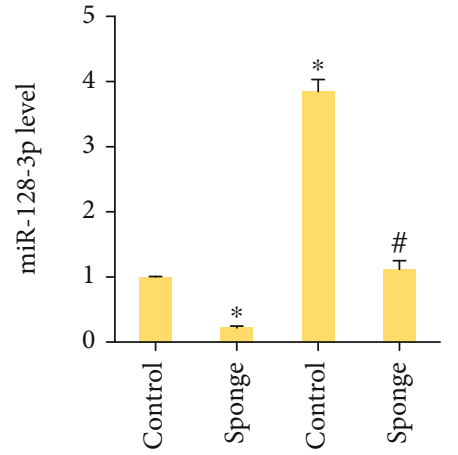

(a)

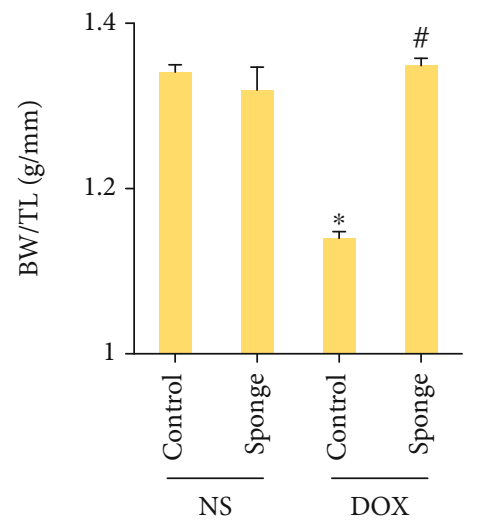

(c)

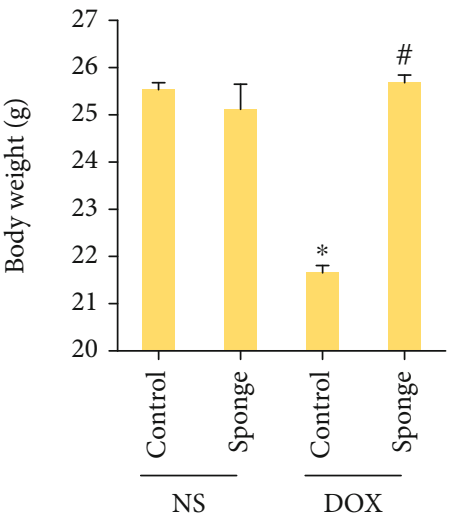

(b)

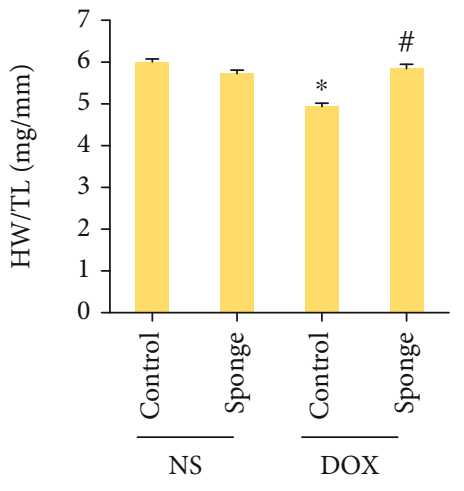

(d)

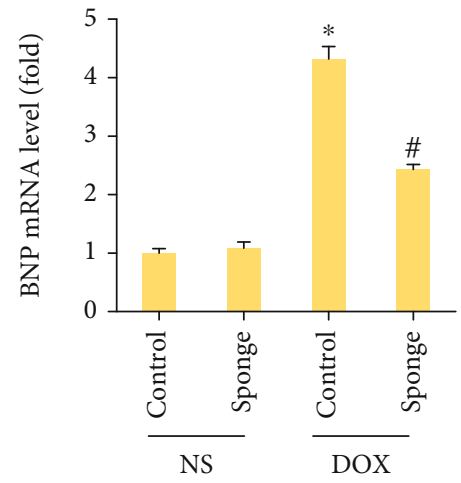

(e)

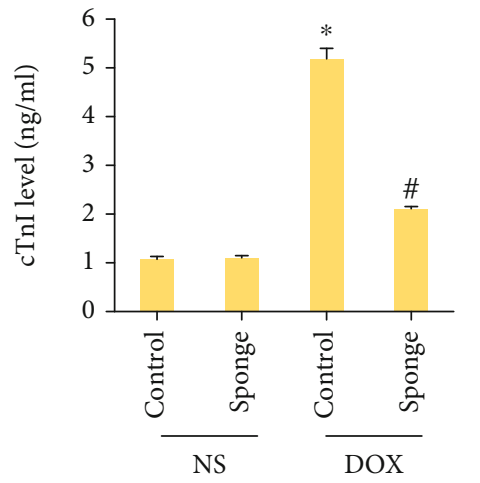

(f)

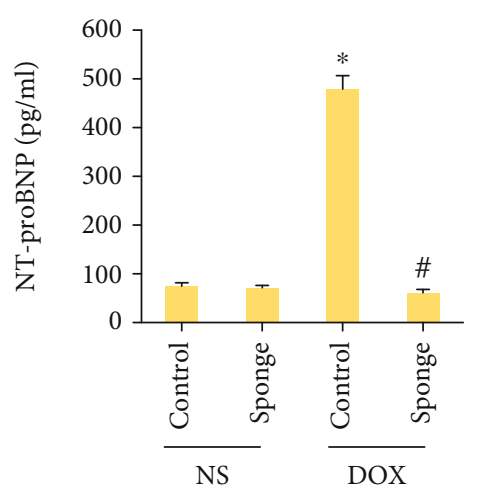

(g)

Figure 2: miR-128-3p inhibition attenuated cardiac injury in DOX-treated mice. (a) The level of miR-128-3p expression in the hearts $(n=6)$. (b) Body weight $(n=12)$. (c) The ratio of body weight to tibial length $(n=12)$. (d) The ratio of heart weight to tibial length $(n=12)$. (e) The mRNA level of BNP in the hearts $(n=6)$. (f, g) The plasma levels of cTnI and NT-proBNP $(n=6)$. Data are expressed as mean \pm SEM. ${ }^{*} P<0.05$ vs. NS/control group; ${ }^{\#} P<0.05$ vs. DOX/control group.

$\mathrm{EF},+\mathrm{dP} / \mathrm{dt}$, and $-\mathrm{dP} / \mathrm{dt}$ were significantly decreased in the DOX group compared with the control group, and miR128-3p inhibition blocked these pathological alterations (Figures 3(b)-3(d)). The increased LVEDP after DOX were suppressed after miR-128-3p inhibition (Figure 3(e)). Taken together, miR-128-3p inhibition attenuated DOX-related acute cardiac injury in mice.

3.3. $m i R-128-3 p$ Inhibition Suppressed Oxidative Damage in DOX-Stimulated Hearts. We assessed the total SOD activity, catalase activity, and Gpx activity and found these were decreased after DOX treatment. And these reductions were blocked after miR-128-3p inhibition (Figures 4(a)-4(c)). miR-128-3p inhibition also increased GSH content in the heart after DOX injection (Figure 4(d)). As shown in Figures 4(e)-4(g), miR-128-3p inhibition significantly reduced MDA, 4-HNE, and 3-NT levels induced by DOX in the hearts. Together, we found that miR-128-3p inhibition in the hearts prevented myocardial oxidative damage in DOX-injected mice. 


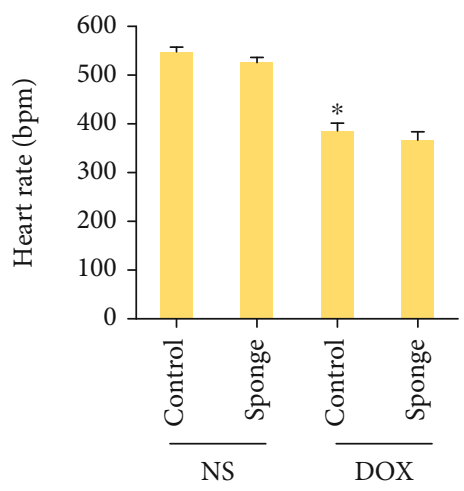

(a)

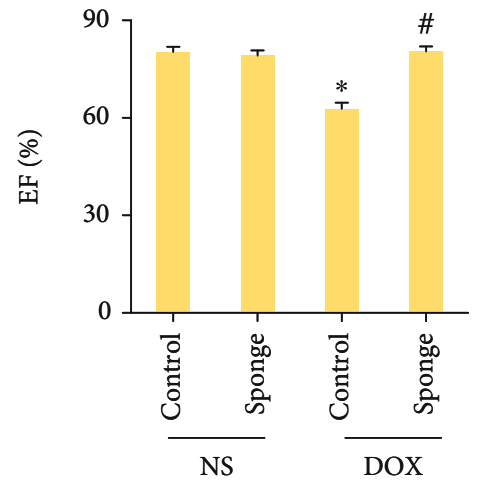

(b)

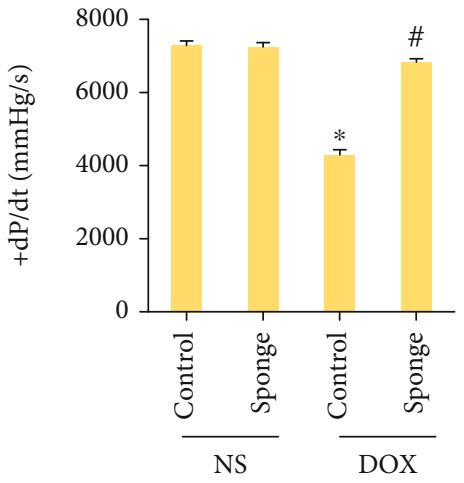

(c)

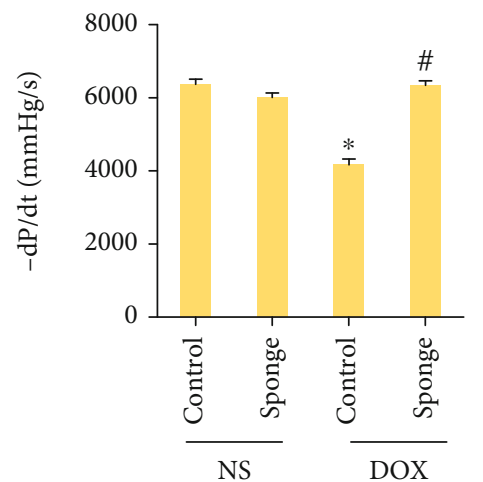

(d)

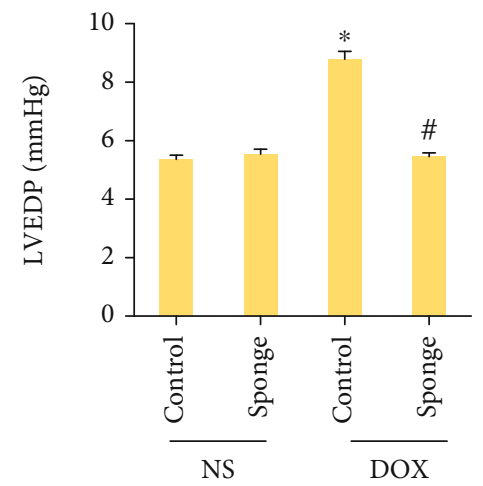

(e)

FIGURE 3: miR-128-3p inhibition improved cardiac function in DOX-treated mice. (a) Heart rate in the indicated groups ( $n=8$ ). (b) Ejection fraction in the indicated groups $(n=8) .(\mathrm{c}, \mathrm{d}) \pm \mathrm{dP} / \mathrm{dt}$ in the mice $(n=8)$. (e) LVEDP in the indicated groups $(n=8)$. Data are expressed as mean \pm SEM. ${ }^{*} P<0.05$ vs. NS/control group; ${ }^{\#} P<0.05$ vs. DOX/control group.

3.4. miR-128-3p Inhibition Suppressed DOX-Induced Inflammatory Response and Apoptosis. To assess the inflammatory response after miR-128-3p inhibition, we first detected p65-DNA binding activity and found that miR128-3p inhibition largely suppressed p65-DNA binding activity in DOX-treated hearts (Figure 5(a)). The myocardial expression of inflammatory cytokines including TNF- $\alpha$ and IL-6 was significantly increased in the model group and was suppressed after miR-128-3p inhibition (Figures 5(b) and 5(c)). Moreover, compared with the control group, nuclear p65 protein expression was increased in DOX group, and the increased nuclear p65 protein expression was decreased by miR-128-3p inhibition (Figures 5(d) and 5(e)). DOX upregulated Bax protein expression, and this upregulation was prevented by the inhibition of miR-128-3p (Figure 5(f)). The increased caspase 3 activity caused by DOX injection was also prevented by the inhibition of miR-128-3p (Figure 5(g)). In addition, after treatment with DOX, an increase in the number of TUNEL-positive cells was observed in DOX-treated mice, and the inhibition of miR-128-3p could decrease the number of these TUNEL-positive cells (Figure 5(h)). Together, these data clearly suggested that miR-128-3p inhibition attenuated inflammation and apoptosis in DOX-treated hearts.

3.5. PPAR- $\gamma$ Was the Target of $m i R-128-3 p$. Using a bioinformatics software, we found that the $3^{\prime}$-UTR of PPAR- $\gamma$ had the binding site with miR-128-3p (Figure 6(a)). As shown in Figure 6(b), transfection of miR-128-3p mimic reduced the luciferase activity of PPAR- $\gamma 3^{\prime}$-UTR in comparison with the negative control group. Further detection revealed that miR-128-3p mimic decreased PPAR- $\gamma$ mRNA and protein expression while the miR-128-3p inhibitor increased PPAR- $\gamma$ mRNA and protein expression (Figures 6(c)-6(f)). We also found miR-128-3p inhibition restored PPAR- $\gamma$ protein expression in DOX-treated hearts (Figure 6(g)). To verify the contribution of PPAR- $\gamma$ to the protection provided by miR-128-3p inhibition, we used a PPAR- $\gamma$ inhibitor. As expected, GW9662 decreased the protein expression of PPAR- $\gamma$ in vitro (Figure 6(h)). GW9662 abolished the protection of miR-128-3p inhibition against p65 activation, TNF- $\alpha$ production, ROS production, and cell loss in response to DOX treatment (Figures 6(i)-6(l)). miR-128-3p inhibition decreased the expression of Bax in DOX-treated cells, and this effect was offset by the use of GW9662 (Figure 6(k)). These data suggested that miR-128-3p inhibition-mediated protection was dependent on the upregulation of PPAR- $\gamma$.

3.6. GW9662 Antagonized the Protective Role of miR-128-3p Inhibition in Mice. To further confirm the role of PPAR- $\gamma$ in the protection against DOX-related cardiac injury, mice were subjected to GW9662 treatment for the inhibition of PPAR- $\gamma$. In line with the finding in vitro, GW9662 decreased myocardial PPAR- $\gamma$ protein expression in mice (Figure $7(\mathrm{a})$ ). The data in our study demonstrated that GW9662 abolished 


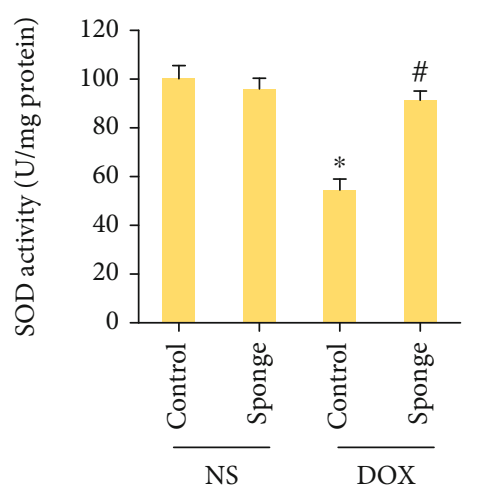

(a)

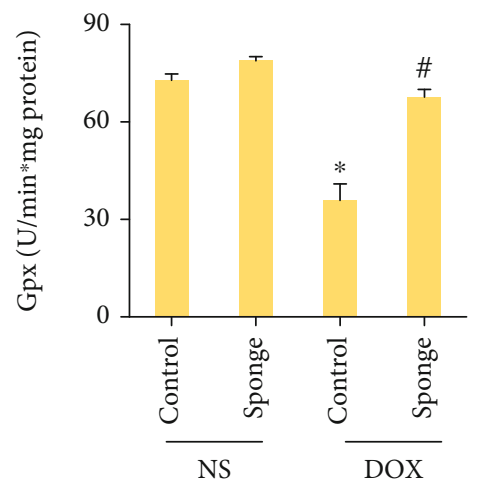

(c)

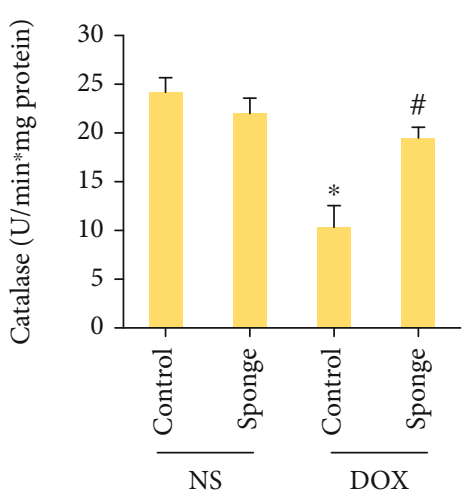

(b)

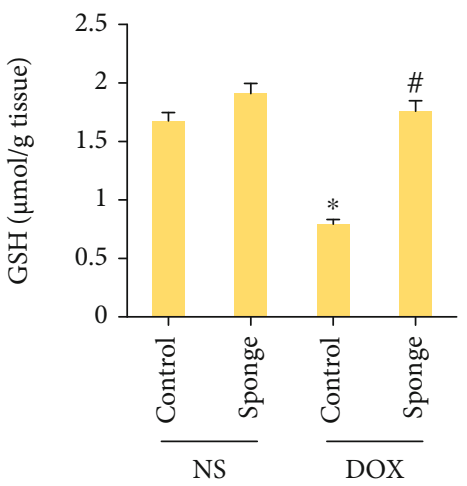

(d)

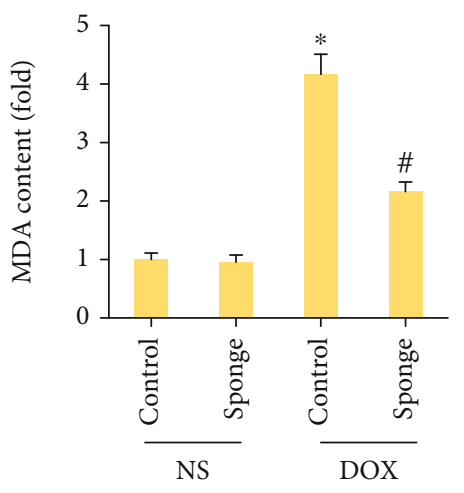

(e)

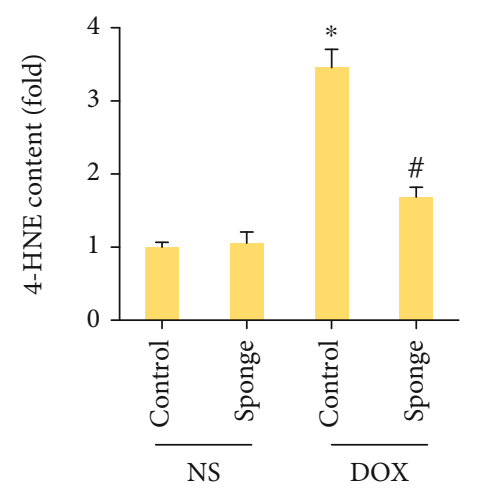

(f)

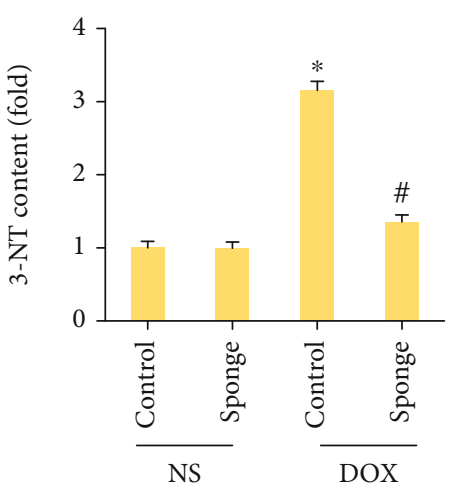

(g)

FIGURE 4: miR-128-3p inhibition attenuated oxidative damage in DOX-treated mice. (a-c) The activities of SOD, catalase, and Gpx in the mice $(n=6)$. (d) The levels of GSH in mice $(n=6)$. (e-g) The levels of MDA, 4-HNE, and 3-NT in the indicated groups $(n=6)$. Data are expressed as mean \pm SEM. ${ }^{*} P<0.05$ vs. NS/control group; ${ }^{\#} P<0.05$ vs. DOX/control group.

the protection in EF, TNF- $\alpha$ production, 4 -HNE production, and caspase 3 activity provided by miR-128-3p inhibition in response to DOX stimuli (Figures 7(b)-7(e)). EF was decreased in response to DOX injection, but improved after the miR-128-3p sponge treatment. This protective effect was also blocked by inhibition of PPAR- $\gamma$ with GW9662 in mice (Figure 7(b)). The increased TNF- $\alpha$ mRNA level, 4HNE production, and caspase 3 activity in DOX-treated hearts were suppressed by the miR-128-3p sponge treatment, and these inhibitory effects were reversed by the treatment of PPAR- $\gamma$ (Figures 7(c)-7(e)). This miR-128-3p sponge treatment decreased myocardial Bax protein expression in
DOX-treated mice, and this effect was offset by the use of GW9662 (Figure 6(k)).

\section{Discussion}

Here, we for the first time demonstrated that DOX treatment increased miR-128-3p expression in murine hearts and cardiomyocytes. Using a miR-128-3p sponge, we found miR128-3p inhibition attenuated DOX-induced cardiac injury and dysfunction in mice and suppressed myocardial oxidative and inflammatory damage, thus improving cardiac function in mice. Further analysis found that miR-128-38 


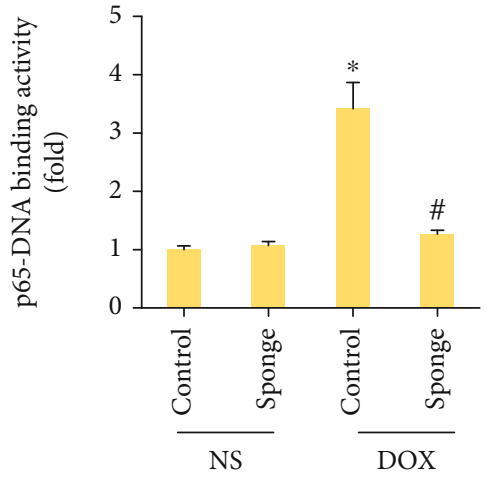

(a)

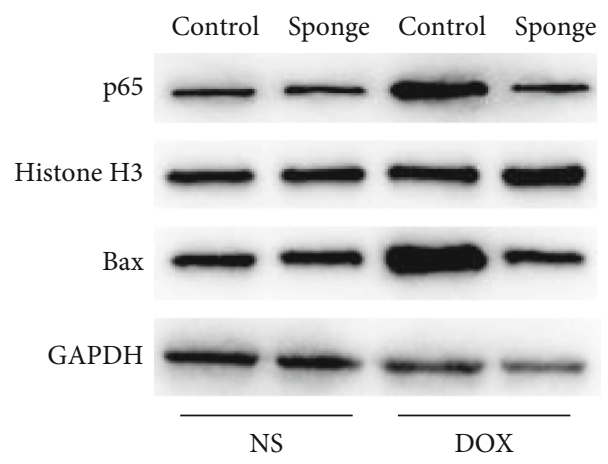

(d)

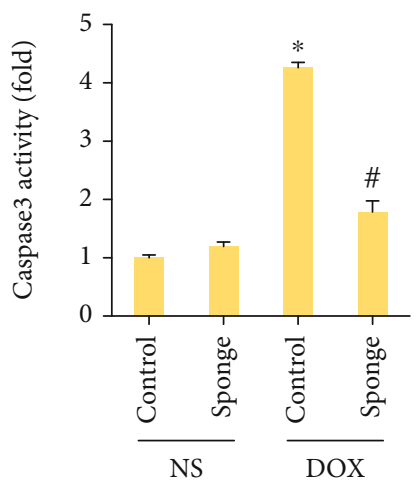

(g)

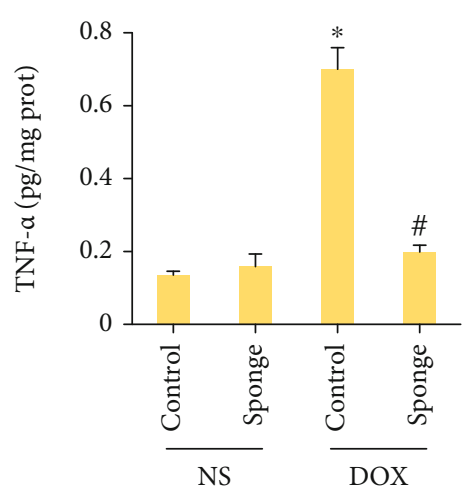

(b)

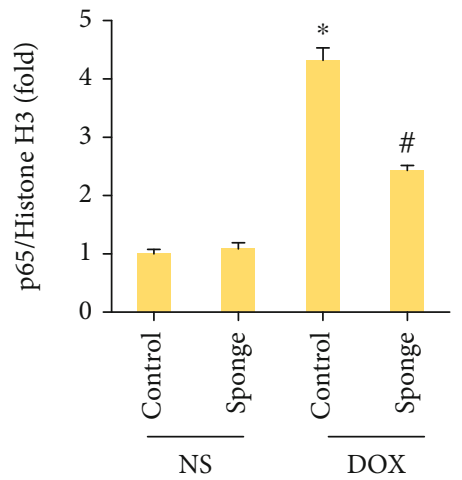

(e)
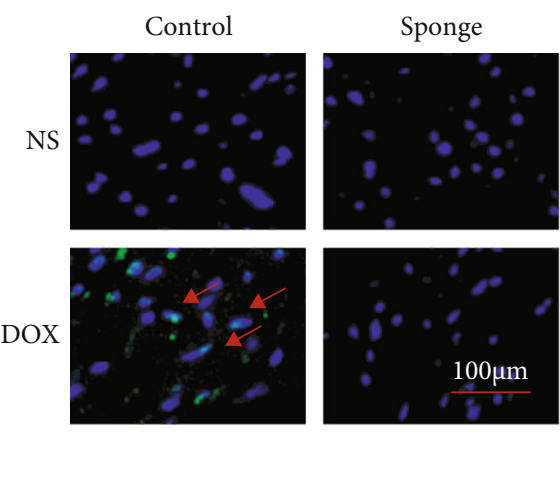

(h)

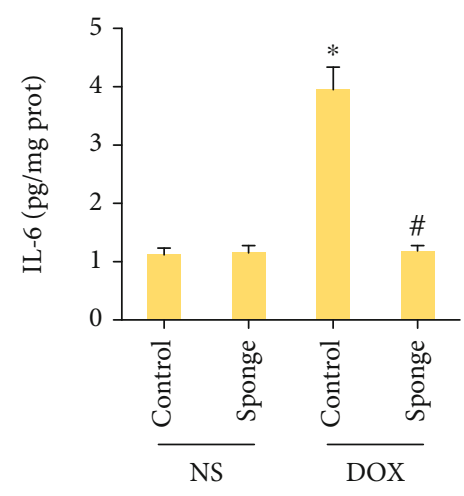

(c)

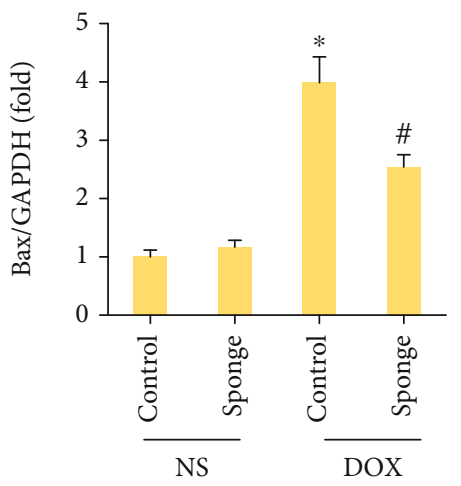

(f)

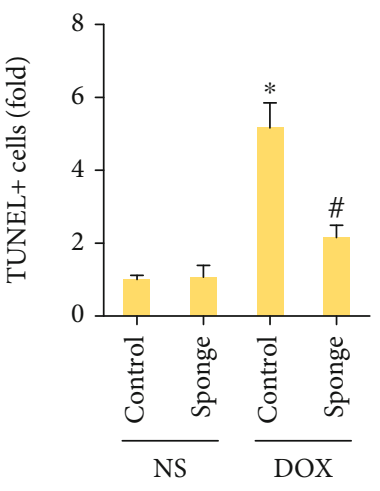

Figure 5: miR-128-3p inhibition attenuated inflammatory response in mice. (a) p65-DNA binding activity $(n=6)$. (b, c) The levels of inflammatory factors in mice $(n=6)$. (d-f) Western blot of Bax and nuclear p65 $(n=6)$. (g) The caspase 3 activity $(n=6)$. (h) TUNEL staining $(n=6)$. Data are expressed as mean \pm SEM. ${ }^{*} P<0.05$ vs. NS/control group; ${ }^{\#} P<0.05$ vs. DOX/control group.

inhibition increased PPAR- $\gamma$ protein expression, and PPAR$\gamma$ inhibition blocked the protection provided by miR-128-38 inhibition in mice. Our data suggested that miR-128-3p inhibition may be a promising approach to treat DOX-related cardiac injury.

It has been reported that miR-128-3p expression was decreased in human heart samples with atrial fibrillation [19]. Chen et al. found that the stimuli of hypoxia and reoxygenation did not affect miR-128-3p expression in human cardiomyocytes [11]. Inconsistent with these studies, miR128-3p was found to be increased in infarcted hearts [20]. Here, we also found that DOX dose- and time-dependently increased miR-128-3p in murine hearts and cardiomyocytes, implying that miR-128-3p was involved into DOX-related cardiac injury. As expected, miR-128-38 inhibition prevented DOX-related cardiac injury, which was in agreement with the finding that inhibition of miR-128-3p by Tongxinluo protected human cardiomyocytes from ischemia/reperfusion injury [11].

Acute DOX injection significantly increased the production of ROS and oxidative products [21]. Moreover, DOXinduced cardiotoxicity could be reduced by the overexpression of the antioxidant enzyme manganese metallothionein [22]. It has been suggested that miRNA-128-3p promoted DOX-induced liver oxidative stress in mice [23]. The data in our study suggested that inhibition of miR-128-3p 


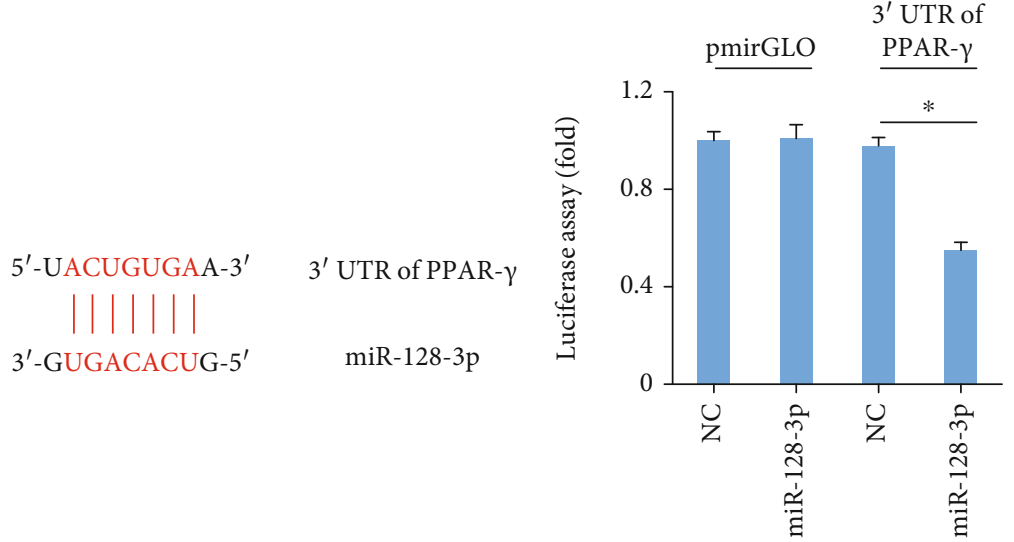

(b)

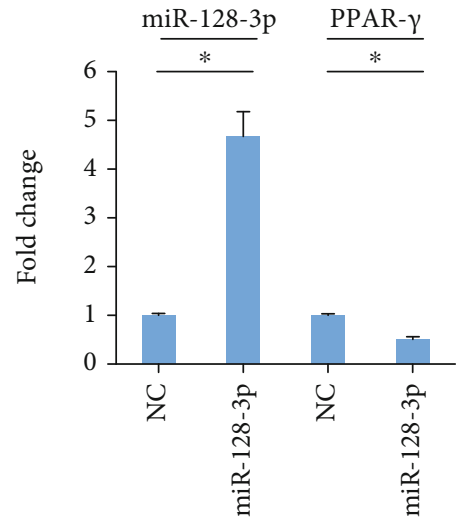

(c)

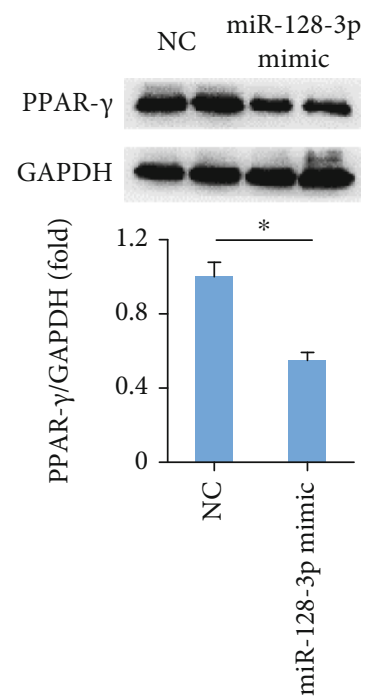

(d)

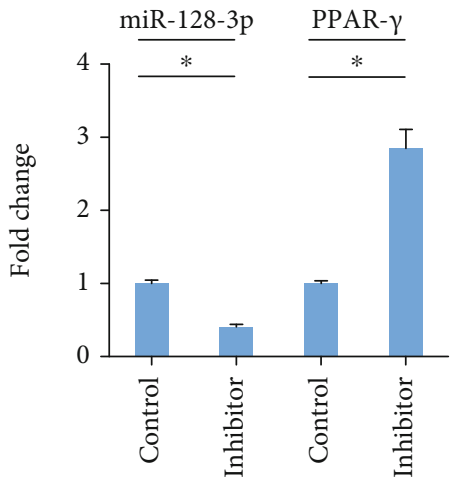

(e)

(f)

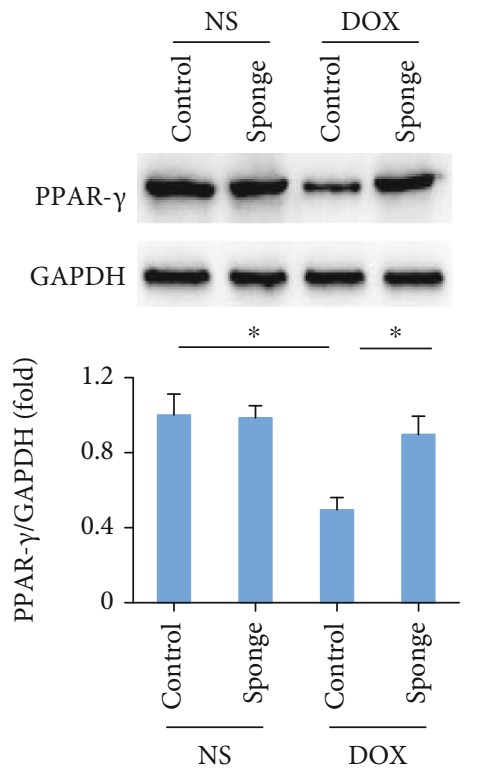

(g)

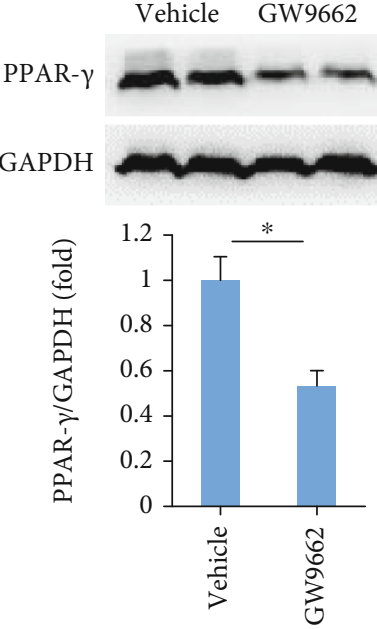

(h)

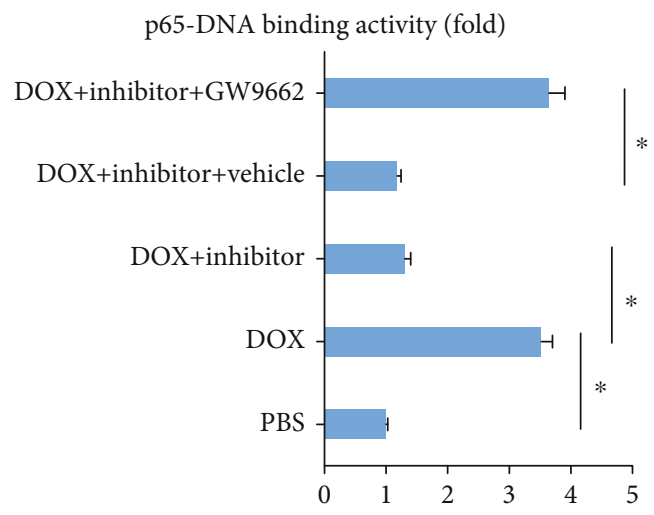

(i)

Figure 6: Continued. 


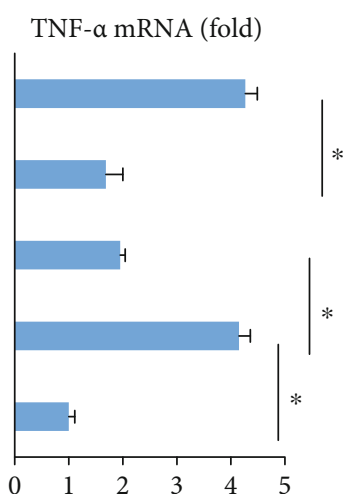

(j)

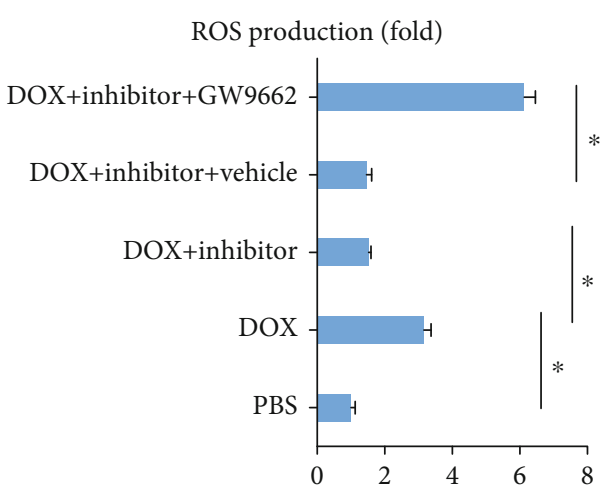

$(\mathrm{k})$

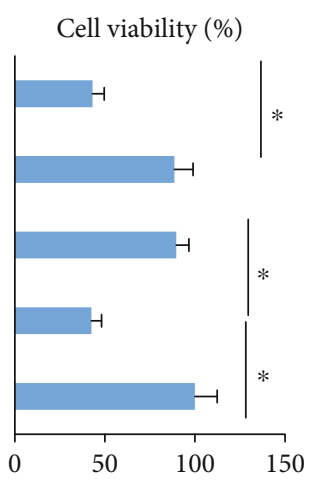

(l)
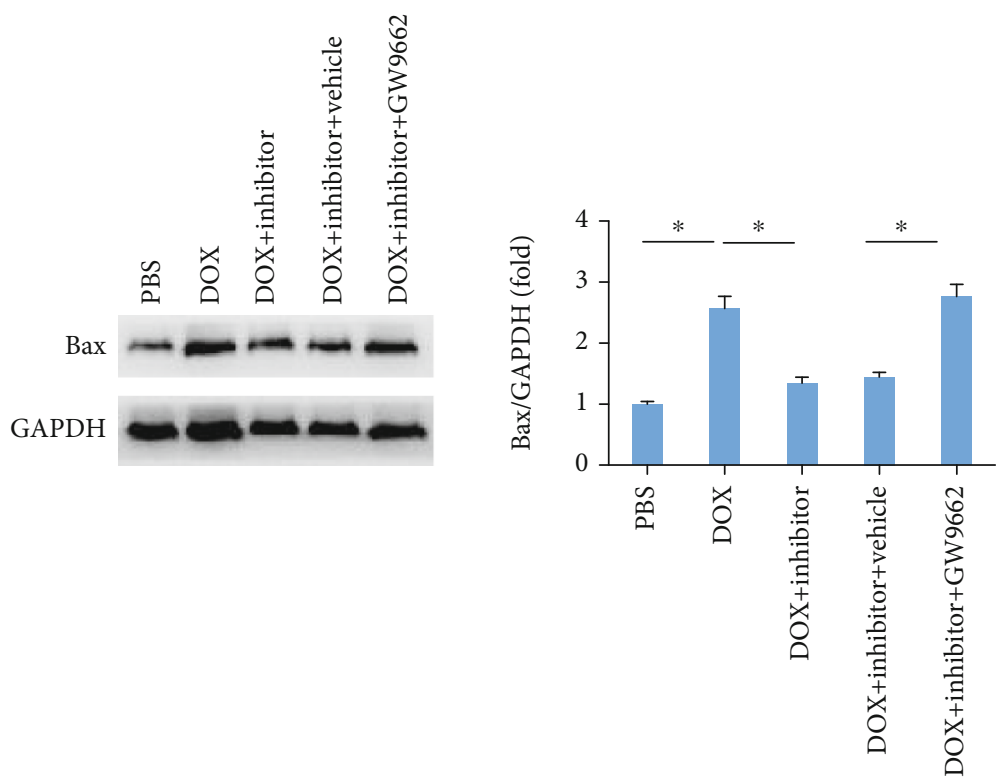

$(\mathrm{m})$

FIGURE 6: miR-128-3p targeted PPAR- $\gamma$ in cardiomyocytes. (a) Binding site with miR-128-3p in $3^{\prime}$-UTR of PPAR- $\gamma$. (b) The luciferase assay $(n=6)$. (c-f) The expression of miR-128-3p and PPAR- $\gamma(n=6)$. (g, h) The protein expression of PPAR- $\gamma(n=6)$. (i) p65-DNA binding activity $(n=6)$. (j) TNF- $\alpha$ mRNA level $(n=6)$. (k) ROS production in the cells $(n=6)$. (l) Cell viability $(n=6)$. (m) Western blot of Bax $(n=6)$. Data are expressed as mean \pm SEM. ${ }^{*} P<0.05$ vs. matched control.

markedly reduced ROS production in DOX-treated cardiomyocytes, reduced MDA, 4-HNE, and 3-NT content, and improved SOD and Gpx activity. The protection by repression of miR-128-3p was partly mediated by the attenuation of oxidative damage.

Although the specific mechanisms of DOX-induced cardiac injury are unclear, increasing evidence has demonstrated that inflammation played an important role in this progression [24]. Of note, the activation of p65 is essential for the development of DOX-mediated cardiotoxicity, and inhibition of p65 could attenuate DOX-induced cardiotoxicity $[25,26]$. A previous study found that repression of miR-128-3p could alleviate liver injury through the regulation of p65 [27]. Here, we also found that repression of miR-128-3p reduced p65 activity and expression, and decreased myocardial inflammation levels in DOX-treated hearts. Taken together, the protection by repression of miR-128-3p was partly mediated by the attenuation of inflammatory response.
Apoptotic cell death is a key component in DOX-induced cardiac dysfunction. DOX treatment resulted in caspase- 3 activation and apoptosis [28, 29]. Moreover, inhibition of DOX-related apoptosis largely attenuated DOX-related cardiac injury [2]. Here, we also found that repression of miR128-3p attenuated DOX-induced cardiac apoptosis in mice and improved cell viability in vitro. The inhibition of cell loss, at least partly, contributed to the protection against DOXrelated injury caused by miR-128-3p depletion.

PPAR- $\gamma$ is a nuclear hormone receptor, which was close in the regulation of lipid metabolism, myocardial health, and embryonic development [30, 31]. Activation of PPAR$\gamma$ could ameliorate cardiac oxidative stress and inflammation in animals with metabolic syndrome and attenuate DOXinduced cardiac injury in mice $[32,33]$, implying that activation of PPAR- $\gamma$ might be an approach to protect from DOXrelated cardiac injury. Here, we found that PPAR- $\gamma$ was a target of miR-128-3p and increased miR-128-3p after DOX treatment decreased PPAR $-\gamma$ expression. Using a sponge, 


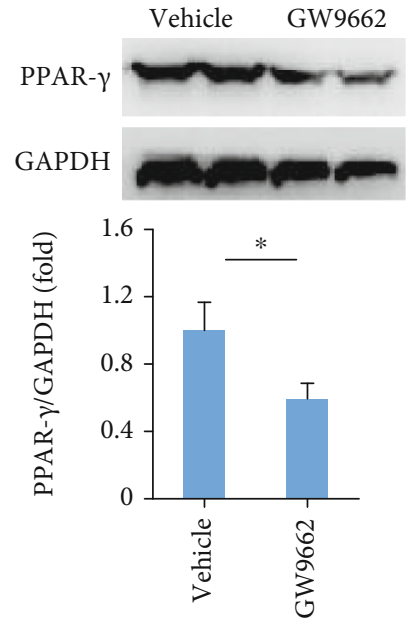

(a)

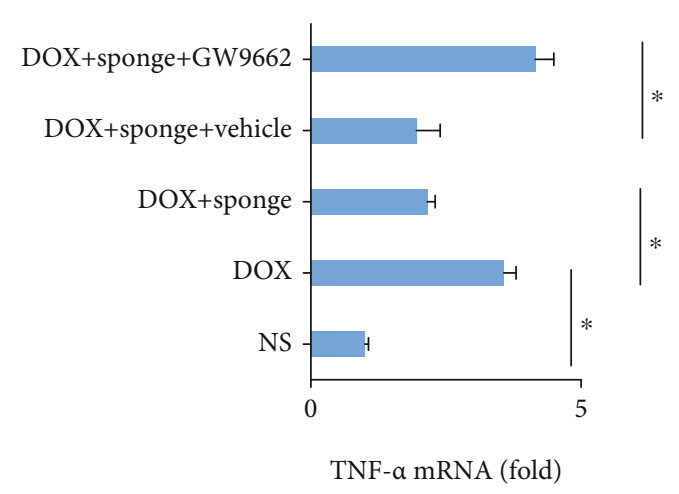

(c)

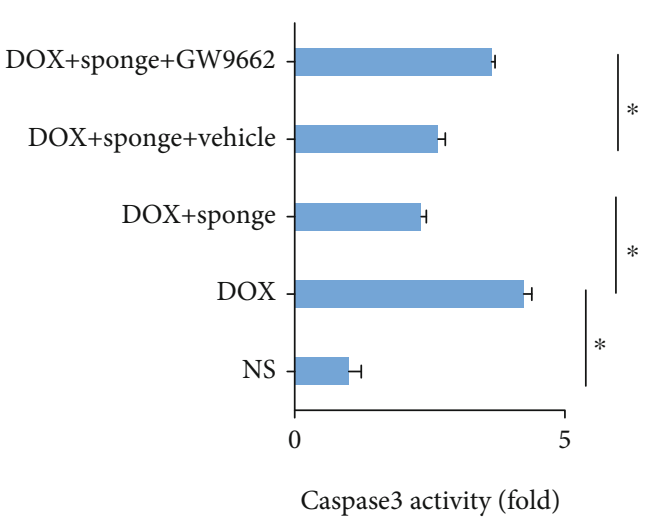

(e)

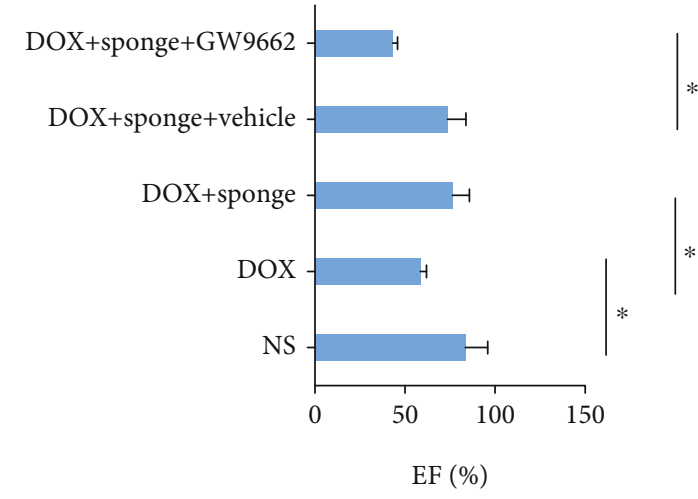

(b)

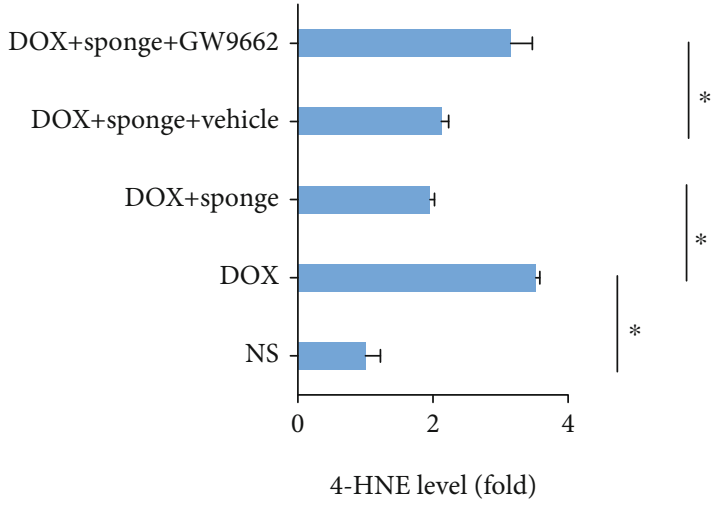

(d)

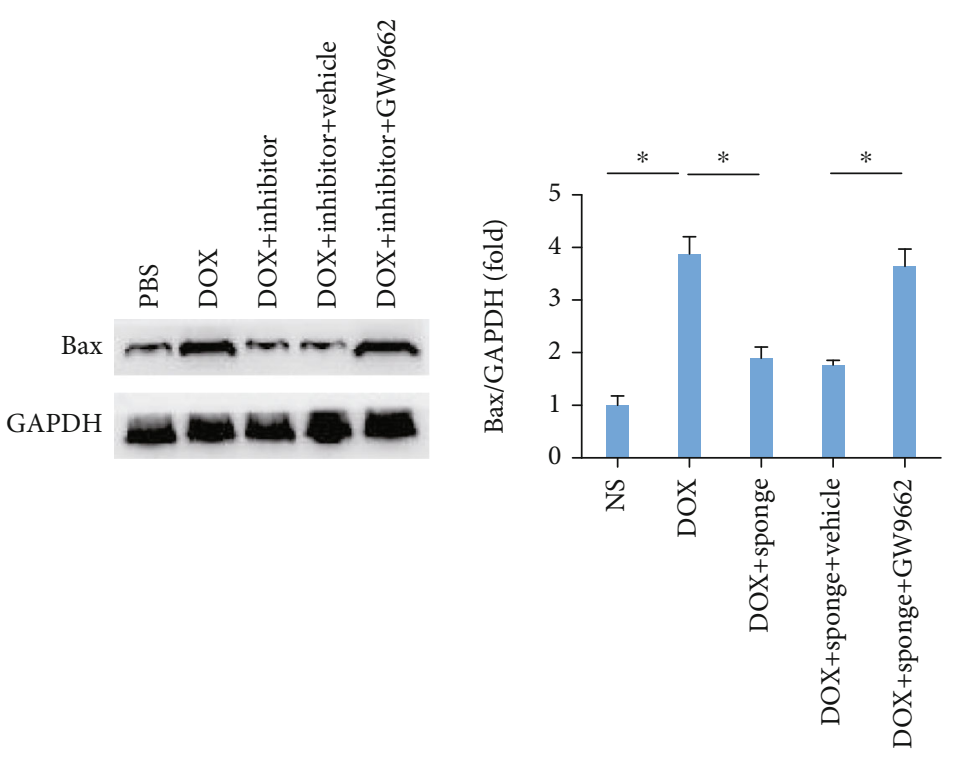

(f)

FIGURE 7: GW9662 abolished protective effects in mice provided by miR-128-3p inhibition. (a) The protein expression of PPAR- $\gamma(n=6)$. (b) Ejection fraction $(n=12)$. (c) TNF- $\alpha$ mRNA level $(n=6)$. (d) 4-HNE level $(n=8)$. (e) Caspase3 activity $(n=8)$. (f) Western blot of Bax $(n=6$ ). Data are expressed as mean \pm SEM. ${ }^{*} P<0.05$ vs. matched control.

we found that miR-128-3p inhibition increased PPAR- $\gamma$ protein expression in DOX-treated hearts. Moreover, these protective effects of miR-128-3p inhibition were blocked by
GW9662 pretreatment, which is an irreversible antagonist of PPAR- $\gamma$, suggesting that miR-128-3p inhibition exerted its cardioprotection via activating PPAR- $\gamma$. 
In conclusion, inhibition of miR-128-3p protected against cardiac injury caused by DOX via activating PPAR$\gamma$ in mice. Inhibition of miR-128-3p may be a promising therapeutic approach to treat chemotherapeutic agentinduced cardiotoxicity.

\section{Data Availability}

The data in our study are available from the corresponding author upon reasonable request.

\section{Conflicts of Interest}

The authors declare that they have no conflicts of interest.

\section{Authors' Contributions}

Wen-bin Zhang and Yong-Fa Zheng contributed equally this work.

\section{Acknowledgments}

This work was supported by grants from the National Natural Science Foundation of China (No. 81703035) and the Fundamental Research Funds for the Central Universities (No. 2042020kf0046).

\section{References}

[1] Y. Octavia, C. G. Tocchetti, K. L. Gabrielson, S. Janssens, H. J. Crijns, and A. L. Moens, "Doxorubicin-induced cardiomyopathy: from molecular mechanisms to therapeutic strategies," Journal of Molecular and Cellular Cardiology, vol. 52, no. 6, pp. 1213-1225, 2012.

[2] Y. P. Yuan, Z. G. Ma, X. Zhang et al., "CTRP3 protected against doxorubicin-induced cardiac dysfunction, inflammation and cell death via activation of Sirt1," Journal of Molecular and Cellular Cardiology, vol. 114, pp. 38-47, 2018.

[3] H. Sawaya, I. A. Sebag, J. C. Plana et al., "Early detection and prediction of cardiotoxicity in chemotherapy-treated patients," The American Journal of Cardiology, vol. 107, no. 9, pp. 1375-1380, 2011.

[4] D. Kumar, L. A. Kirshenbaum, T. Li, I. Danelisen, and P. K. Singal, "Apoptosis in adriamycin cardiomyopathy and its modulation by probucol," Antioxidants \& Redox Signaling, vol. 3, no. 1, pp. 135-145, 2001.

[5] T. Simunek, M. Sterba, O. Popelova, M. Adamcova, R. Hrdina, and V. Gersl, "Anthracycline-induced cardiotoxicity: overview of studies examining the roles of oxidative stress and free cellular iron," Pharmacological Reports, vol. 61, no. 1, pp. 154$171,2009$.

[6] A. C. Childs, S. L. Phaneuf, A. J. Dirks, T. Phillips, and C. Leeuwenburgh, "Doxorubicin treatment in vivo causes cytochrome $\mathrm{C}$ release and cardiomyocyte apoptosis, as well as increased mitochondrial efficiency, superoxide dismutase activity, and Bcl-2:Bax ratio," Cancer Research, vol. 62, no. 16, pp. 4592-4598, 2002.

[7] J. Li, S. X. Cai, Q. He et al., "Intravenous miR-144 reduces left ventricular remodeling after myocardial infarction," Basic Research in Cardiology, vol. 113, no. 5, p. 36, 2018.
[8] Y. Bei, X. Wu, D. Cretoiu et al., "miR-21 suppression prevents cardiac alterations induced by d-galactose and doxorubicin," Journal of Molecular and Cellular Cardiology, vol. 115, pp. 130-141, 2018.

[9] L. Zhao, R. Li, S. Xu et al., "Tumor suppressor miR-128-3p inhibits metastasis and epithelial-mesenchymal transition by targeting ZEB1 in esophageal squamous-cell cancer," Acta Biochimica et Biophysica Sinica, vol. 50, no. 2, pp. 171180, 2018.

[10] J. Liu, S. Wang, Q. Zhang, X. Li, and S. Xu, "Selenomethionine alleviates LPS-induced chicken myocardial inflammation by regulating the miR-128-3p-p38 MAPK axis and oxidative stress," Metallomics, vol. 12, no. 1, pp. 54-64, 2020.

[11] G. H. Chen, C. S. Xu, J. Zhang et al., "Inhibition of miR-128-3p by tongxinluo protects human cardiomyocytes from ischemia/reperfusion injury via upregulation of $\mathrm{p} 70 \mathrm{~s} 6 \mathrm{k} 1 / \mathrm{p}$ p70s6k1," Frontiers in Pharmacology, vol. 8, p. 775, 2017.

[12] X. Zhang, C. Hu, C. Y. Kong et al., "FNDC5 alleviates oxidative stress and cardiomyocyte apoptosis in doxorubicin-induced cardiotoxicity via activating AKT," Cell Death and Differentiation, vol. 27, no. 2, pp. 540-555, 2020.

[13] Z. G. Ma, Y. P. Yuan, X. Zhang et al., "C1q-tumour necrosis factor-related protein-3 exacerbates cardiac hypertrophy in mice," Cardiovascular Research, vol. 115, no. 6, pp. 10671077, 2019.

[14] L. J. Min, M. Mogi, M. Shudou et al., "Peroxisome proliferatoractivated receptor- $\gamma$ activation with angiotensin II type 1 receptor blockade is pivotal for the prevention of blood-brain barrier impairment and cognitive decline in type 2 diabetic mice," Hypertension, vol. 59, no. 5, pp. 1079-1088, 2012.

[15] Z. G. Ma, J. Dai, Y. P. Yuan et al., "T-bet deficiency attenuates cardiac remodelling in rats," Basic Research in Cardiology, vol. 113, no. 3, p. 19, 2018.

[16] Z. G. Ma, Y. P. Yuan, S. C. Xu et al., "CTRP3 attenuates cardiac dysfunction, inflammation, oxidative stress and cell death in diabetic cardiomyopathy in rats," Diabetologia, vol. 60, no. 6, pp. 1126-1137, 2017.

[17] Z. G. Ma, J. Dai, W. B. Zhang et al., "Protection against cardiac hypertrophy by geniposide involves the GLP-1 receptor/AMPK $\alpha$ signalling pathway," British Journal of Pharmacology, vol. 173, no. 9, pp. 1502-1516, 2016.

[18] W. B. Zhang, F. Yang, Y. Wang et al., "Inhibition of HDAC6 attenuates LPS-induced inflammation in macrophages by regulating oxidative stress and suppressing the TLR4-MAPK/NF$\kappa \mathrm{B}$ pathways," Biomedicine \& Pharmacotherapy, vol. 117, p. 109166, 2019.

[19] F. Cao, Z. Li, W. M. Ding, L. Yan, and Q. Y. Zhao, "LncRNA PVT1 regulates atrial fibrosis via miR-128-3p-SP1-TGF- $\beta 1$ Smad axis in atrial fibrillation," Molecular Medicine, vol. 25, no. 1, p. 7, 2019.

[20] H. Ma, P. Chen, C. Sang, D. Huang, Q. Geng, and L. Wang, "Modulation of apoptosis-related microRNAs following myocardial infarction in fat-1 transgenic mice vs wild-type mice," Journal of Cellular and Molecular Medicine, vol. 22, no. 11, pp. 5698-5707, 2018.

[21] M. Songbo, H. Lang, C. Xinyong, X. Bin, Z. Ping, and S. Liang, "Oxidative stress injury in doxorubicin-induced cardiotoxicity,” Toxicology Letters, vol. 307, pp. 41-48, 2019.

[22] Y. J. Kang, Y. Chen, A. Yu, M. Voss-McCowan, and P. N. Epstein, "Overexpression of metallothionein in the heart of transgenic mice suppresses doxorubicin cardiotoxicity," The 
Journal of Clinical Investigation, vol. 100, no. 6, pp. 1501-1506, 1997.

[23] X. Zhao, Y. Jin, L. Li et al., "MicroRNA-128-3p aggravates doxorubicin-induced liver injury by promoting oxidative stress via targeting Sirtuin-1," Pharmacological Research, vol. 146, p. 104276, 2019.

[24] J. Ye, Y. Huang, B. Que et al., "Interleukin-12p35 knock out aggravates doxorubicin-induced cardiac injury and dysfunction by aggravating the inflammatory response, oxidative stress, apoptosis and autophagy in mice," EBioMedicine, vol. 35, pp. 29-39, 2018.

[25] K. Ashikawa, S. Shishodia, I. Fokt, W. Priebe, and B. B. Aggarwal, "Evidence that activation of nuclear factor-kappaB is essential for the cytotoxic effects of doxorubicin and its analogues," Biochemical Pharmacology, vol. 67, no. 2, pp. 353364, 2004.

[26] J. Han, Y. Jun, S. H. Kim et al., "Rapid emergence and mechanisms of resistance by U87 glioblastoma cells to doxorubicin in an in vitro tumor microfluidic ecology," Proceedings of the National Academy of Sciences of the United States of America, vol. 113, no. 50, pp. 14283-14288, 2016.

[27] T. Mou, Y. Luo, Z. Huang et al., "Inhibition of microRNA-128$3 p$ alleviates liver ischaemia-reperfusion injury in mice through repressing the Rnd $3 / \mathrm{NF}-\kappa \mathrm{B}$ axis," Innate Immunity, vol. 26, no. 6, pp. 528-536, 2020.

[28] J. N. Zhu, Y. H. Fu, Z. Q. Hu et al., "Activation of miR-34a$5 \mathrm{p} /$ Sirt1/p66shc pathway contributes to doxorubicin-induced cardiotoxicity," Scientific Reports, vol. 7, no. 1, p. 11879, 2017.

[29] M. Kuzu, F. M. Kandemir, S. Yildirim, S. Kucukler, C. Caglayan, and E. Turk, "Morin attenuates doxorubicininduced heart and brain damage by reducing oxidative stress, inflammation and apoptosis," Biomedicine \& Pharmacotherapy, vol. 106, pp. 443-453, 2018.

[30] D. P. Foti, F. Paonessa, E. Chiefari, and A. Brunetti, "New Target Genes for the Peroxisome Proliferator-Activated Receptor(PPAR) Antitumour Activity: Perspectives from the Insulin Receptor," PPAR Research, vol. 2009, Article ID 571365, 8 pages, 2009.

[31] V. Costa, D. Foti, F. Paonessa et al., "The insulin receptor: a new anticancer target for peroxisome proliferator-activated receptor-gamma (PPARgamma) and thiazolidinedionePPARgamma agonists," Endocrine-Related Cancer, vol. 15, no. 1, pp. 325-335, 2008.

[32] S. Arunachalam, P. B. Tirupathi Pichiah, and S. Achiraman, "Doxorubicin treatment inhibits PPAR $\gamma$ and may induce lipotoxicity by mimicking a type 2 diabetes-like condition in rodent models," FEBS Letters, vol. 587, no. 2, pp. 105-110, 2013.

[33] G. Pakravan, A. M. Foroughmand, M. Peymani et al., "Downregulation of miR-130a, antagonized doxorubicin-induced cardiotoxicity via increasing the _PPAR $~ \gamma$ expression in mESCs-derived cardiac cells," Cell Death \& Disease, vol. 9, no. 7 , p. $758,2018$. 